\title{
Detection of electric treeing of solid dielectrics with the method of acoustic emission
}

\author{
Władysław Opydo • Arkadiusz Dobrzycki
}

Received: 31 August 2009 / Accepted: 5 June 2011 / Published online: 19 June 2011

(C) The Author(s) 2011. This article is published with open access at Springerlink.com

\begin{abstract}
The paper presents results of research of electrical treeing of solid dielectrics with the method of acoustic emission (AE). The study was performed with an alternating voltage of $50 \mathrm{~Hz}$ in the range up to $21 \mathrm{kV}$ (RMS) on methyl polymethacrylate or crosslinked polyethylene samples. They were of cuboidal shape of the dimensions $25 \times 10 \times 4 \mathrm{~mm}$. One of the cuboid sample walls of the dimensions $25 \times 4 \mathrm{~mm}$ was covered with a conducting paint. On the opposite wall, a surgical needle of T-25 type was screwed. The distance between the electrodes (the needle and the wall covered with a conducting paint) was in the range $0.5-2.0 \mathrm{~mm}$. Registered signals were denoised with wavelet transformation method and then there were analyzed. The following parameters were analyzed: a sum and rate of acoustic emission counting, a sum and rate of acoustic emission events, RMS value of the electric signal leaving the converter. Spectrum and spectrogram were also analyzed. It was found that AE signals are generated during electrical treeing of solid dielectrics. Values of chosen parameters increased their values when the process begins. There are also some dominant frequencies ranges, different for different kinds of dielectrics, connected with the treeing.
\end{abstract}

Keywords Acoustic emission - Electrical treeing . Electrical insulation $\cdot$ Solid dielectrics

\section{Introduction}

Permanent growth of electric power demand, particularly high in large industrial and urban centers, induces the use of

W. Opydo · A. Dobrzycki $(\bowtie)$

Institute of Electrical Engineering and Electronics,

Poznan University of Technology, Piotrowo 3A,

Poznan 60-965, Poland

e-mail: arkadiusz.dobrzycki@put.poznan.pl higher and higher voltage power networks for transmission of electric power. At the same time, decreasing quantity of free building areas in these centers force the use of electric power cables and miniaturization of high-voltage network equipment. These two factors cause that the electric power devices must operate under higher intensity of the electric field. Therefore, their high-voltage insulation must be more perfect.

In an electric-power device, particularly, the one designed for high voltage operation, there occurs a problem of electric strength of solid materials. Any electric or electric-power device, even the one in which gas, vacuum, or mineral oil is a basic electric insulation, must include a solid dielectric. It enables fixing to the casing the elements subject to high voltage. Moreover, it allows for inputting the electric power to and outputting it from the casing or construction, having usually the earth potential (the lead-in insulators). Hence, any high-voltage electric or electric-power device give rise to the problem of electric strength of the solid dielectrics used as their construction materials.

In high-voltage equipment with solid dielectrics the main destructive factor is related to partial discharges (PD) occurring at their surfaces or inside the structure. PD occurrence causes worsening of the electro-insulation properties and is conducive to progressing destruction of the insulation. PD in dielectrics is frequently related to electric treeing, consisting in generation of conductive channels inside the dielectric material. The discharges occurring in these channels induce further treeing process, leading to a tree or bush-like form, finally causing shortcircuiting of the electrodes. Thus, for the insulation subject to treeing, it is a catastrophic forecast. Therefore, diagnosis of the high-voltage insulation is significantly affected by the information whether the treeing process is already in course. 
Fig. 1 Measurement stand schema (a) and photo of the sample in a holder (b)

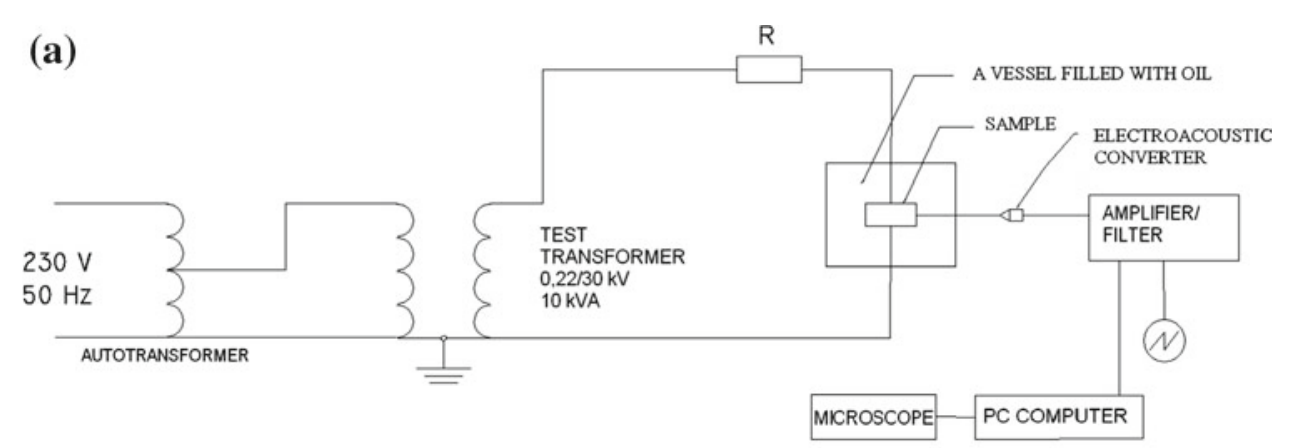

(b)

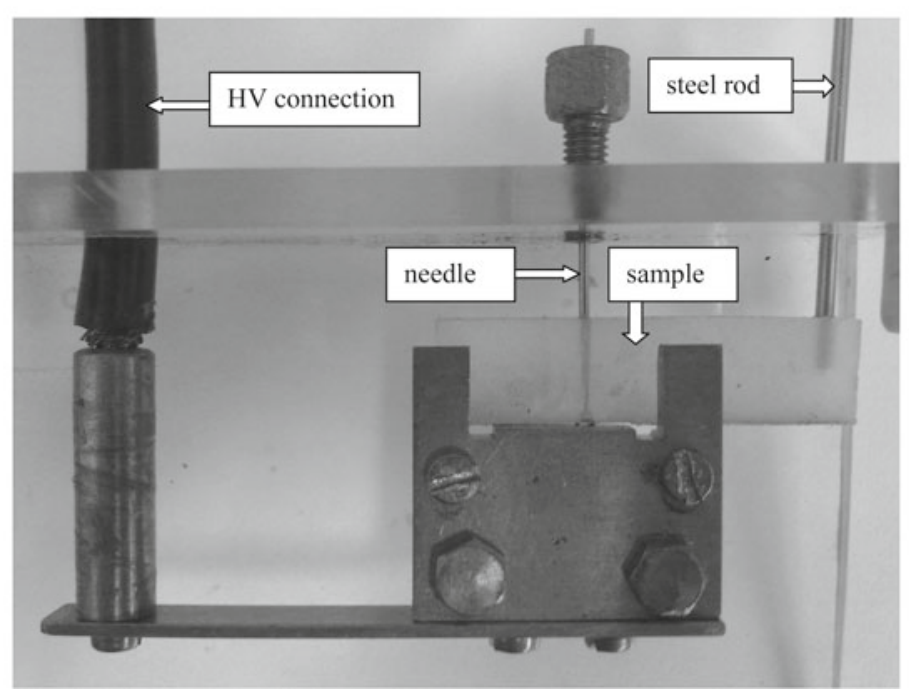

The investigation related to the above topic is related, among others, to the description of PD characteristics that is related to the electric treeing process $[10,11]$. Special attention is also paid to forecast the treeing during its propagation $[2-4,9,12,14]$. The studies, mainly of simulation character, were aimed at probable shape and rate of the tree development in the considered insulation system. These works make use of highly advanced mathematical analysis - the theories of fractals and deterministic chaos.

The present paper deals with the problem of detecting the treeing process undergoing in solid dielectrics subject to electric stress. Detection of the process is performed with the acoustic emission method. It was found $[6,7]$ that electric treeing of solid dielectrics is accompanied by emission of elastic waves of acoustic frequencies. Appropriately sensitive devices may detect the waves. Advantage of the method consists in its application during operation of the device, without interruption of the customers' supply. It should be noticed that the term of acoustic emission method is not accurate, as the signals of the frequency range from several $\mathrm{mHz}$ to one $\mathrm{GHz}$ are used in it, hence the ones laying beyond the acoustic range.

\section{Description of the study and the measuring stand}

The study has been performed on methyl polymethacrylate or crosslinked polyethylene samples. They were of cuboidal shape of the dimensions $25 \times 10 \times 4 \mathrm{~mm}$. One of the cuboid sample walls of the dimensions $25 \times 4 \mathrm{~mm}$ was covered with a conducting paint.On to the opposite, wall a surgical needle of T-25 type was screwed on and such a needle has a tip with $12 \mu \mathrm{m}$ radius of curvature. The needle previously heated was screwed in under microscope and such a procedure allowed to adjust the distance between the needle tip and the conducting paint layer and, in consequence, the size of dielectric space subject to the electric stress.

The sample prepared this way was located in a transparent cuboid container made of methyl polymethacrylate, filled with transformer oil.

As a voltage source, a test transformer of $220 \mathrm{~V} / 30 \mathrm{kV}$ ratio and $10 \mathrm{kVA}$ was used. It was supplied from a low-voltage distribution network through an adjustment autotransformer. One of the test transformer terminals was connected to the conducting paint layer while the other, through a $R=200 \mathrm{k} \Omega$ resistor to the needle electrode. 


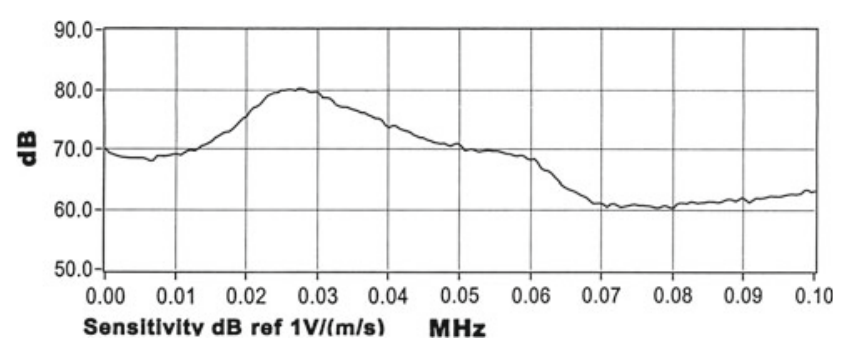

Fig. 2 Characteristics of the electroacoustic converter used for the research purpose [8]

The high voltage was measured with an electrostatic voltmeter.

Measurement stand schema and photo of the sample in a holder is shown on Fig. 1.

The sample located in the oil-filled container has been observed under an STM 723 type microscope with adjusted magnification (from 40 to $160 \times$ ). The microscope was provided with CCD camera of medium resolution. The vision signal from the camera was transmitted to a TV card installed in a computer and, apart from the monitor presentation, was recorded every minute in the form of graphical files.

For purpose of measuring the acoustic emission signals, an R3 $\alpha$-type piezoelectric converter manufactured by PAC has been used. A characteristic of the converter efficiency is shown in Fig. 2. The elastic waves generated in the sample were transmitted to the converter with a wave guide. The wave guide was made of a steel rod of $2 \mathrm{~mm}$ diameter and a length of $20 \mathrm{~cm}$. One of the wave guide ends was located in a hole bored in the dielectric sample (next to the needle, at a distance $2.5 \mathrm{~cm}$ ), while the other was fixed in a cone made of polymethacrylate methyl. The cone was connected to the converter through an acoustic paste and that length is guaranteed not to incite the rod during measurement. The electric signal from the converter was amplified with a 2/4/6 PAC preamplifier and then filtered with a filter of $20-1,000 \mathrm{kHz}$ transmission band. Afterwards, the signal was amplified by an AE5A PAC amplifier and recorded on a computer hard disk with the use of a measuring card of Waveterminal 192X type manufactured by ESI.

While the recording procedure the signal has been sampled with $192 \mathrm{kHz}$ frequency and 16 bits resolution in the voltage range $\pm 1 \mathrm{~V}$.

\section{Description of the research method}

Among many parameters of the acoustic emission signals for purposes of description of the measured values the following ones have been adopted:

- a sum and rate of acoustic emission counting;

- a sum and rate of acoustic emission events;

- RMS value of the electric signal leaving the converter.

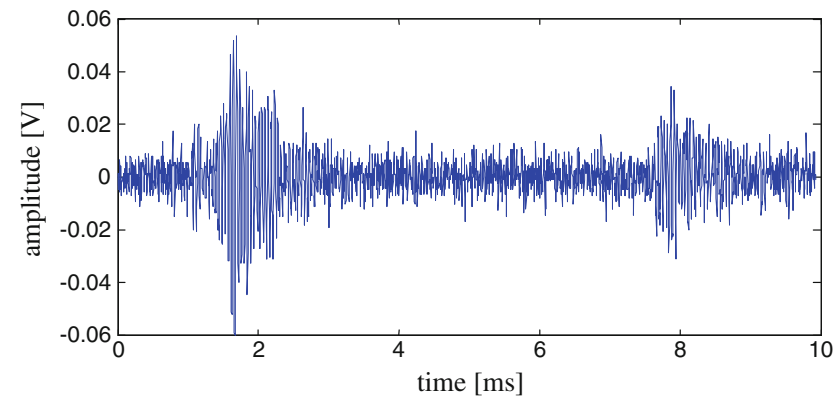

Fig. 3 Example time pattern of the EA signals during the treeing process

The sum of acoustic emission counting ( $\Sigma \mathrm{EA}$ ) is a sum of signal amplitudes exceeding an arbitrarily assumed discrimination threshold, counted in a definite period of time. On the other hand, the counting rate $(\Sigma \mathrm{EA} / \Delta t)$ is a counting sum referred to the measuring time $(\Delta t)$.

The sum of acoustic events $(\Sigma N)$ is defined as the number of the events counted in a definite period of time the envelopes of which (as opposed to the amplitudes as in case of the sum of counting) exceed an arbitrarily assumed discrimination threshold. On the other hand, the rate of the events $(\Sigma N / \Delta t)$ is a sum of the events referred to the measuring time $(\Delta t)$.

The root mean square value $A_{\mathrm{RMS}}$ of the electric signal leaving the electroacoustic converter (briefly called an RMS signal) is defined as

$A_{\mathrm{RMS}}=\sqrt{\frac{1}{T} \int_{0}^{T} u^{2}(t) \mathrm{d} t}$,

where $u(t)$, the voltage time pattern of the converter output; $T$, impulse duration.

The amplitude spectrum of EA signals usually includes the bands of predominant frequencies. Therefore, during the research, a frequency analysis of the measured signals has been made.

As the acoustic signals generated by the samples subject to electric stress were rather low (Fig. 3), the amplitudes of the measured signals might be below the noise amplitudes recorded or generated in the measuring system. This was a reason for developing a method of minimization of the disturbance effect on the measurement results. Although analyzing the values of the descriptors defined as the derivatives of time variations, the characteristic property of the noise has been used consisting in zeroing of its average value [13]. Hence, averaging of the sample set leads to the increase of the signal to noise ratio (in consequence the noise amplitude decreases). In case of analysis of the other descriptors the noise is eliminated from the signal by application of the wavelet transformation [1]. The noise has been eliminated according to the following algorithm: 
Fig. 4 Pictures of the inter-electrode space of the methyl polymethacrylate sample: a start of the measurement, b 3 min after the measurement start, c $20 \mathrm{~min}$ after the measurement start, d 40 min after the measurement start, e 60 min after the measurement start
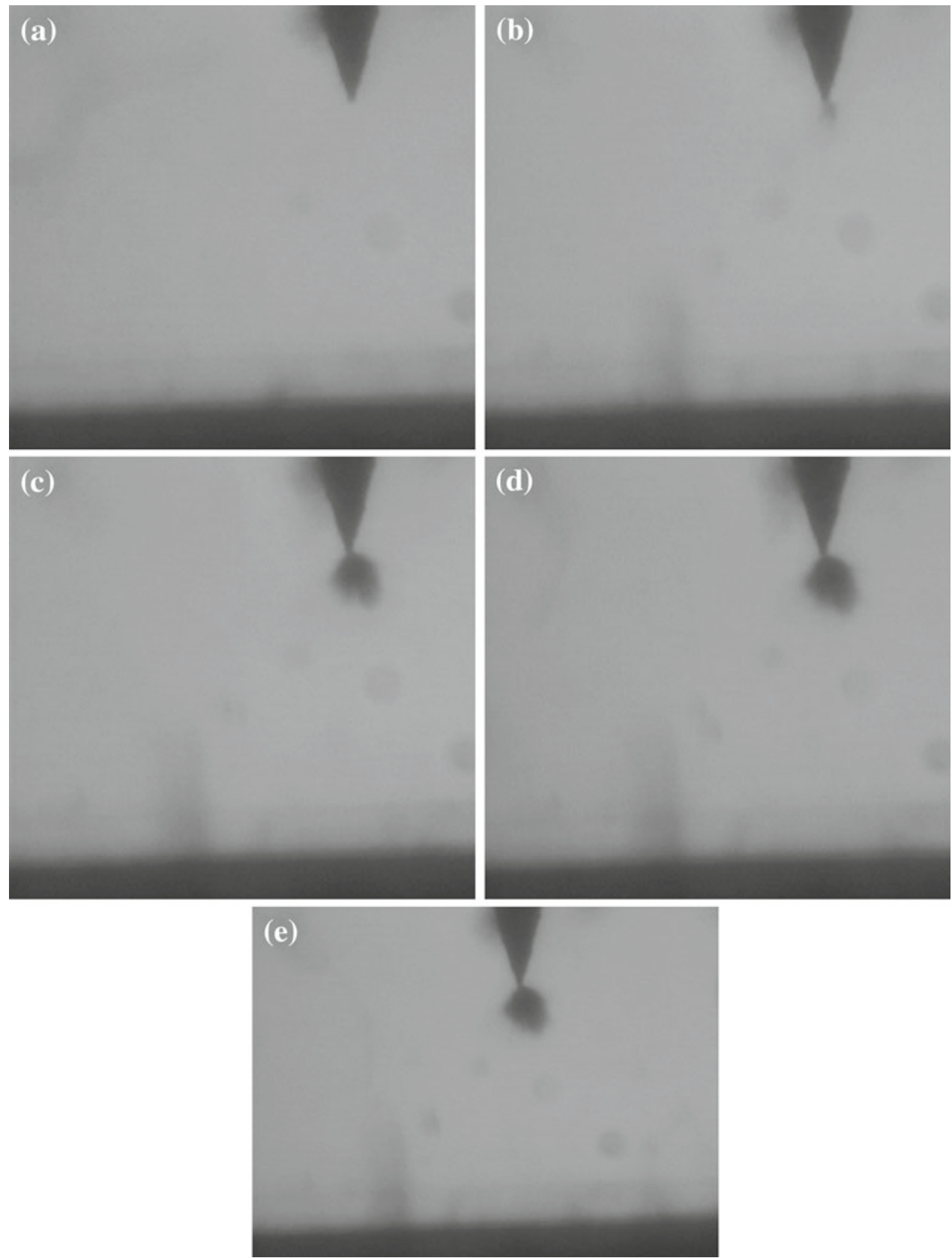

- stage 1: signal decomposition (calculation of wavelet coefficients of the given wavelet);

- stage 2: analysis of threshold values of the wavelet coefficients and their correction ("smoothing");

- stage 3: signal reconstruction based on the modified wavelet coefficients.

The other problem resulted from large size file of the measurement data (above $1 \mathrm{~GB}$ ) collected during the measurement procedure lasting about $1 \mathrm{~h}$. To determine the descriptors being the derivatives of the time-variations a computer program has been developed in the MS Visual Studio environment. The program itself has been formulated in C\#, while the numerical procedures designed to determine the root mean square variations and frequency and timefrequency distributions have been developed in MatLab. Details of the developed programs can be found in [5].

\section{Results of the research and their discussion}

The following are typical results of measurements for each of the tested materials. Different distances between electrodes and different voltage levels, and the various procedures affect the change descriptors in a similar manner. Initiation of 
Fig. 5 Changes in the values of $\mathbf{a}$ the sum, and $\mathbf{b}$ the counting rate of the EA signals during the electric treeing test of the sample made of methyl polymethacrylate
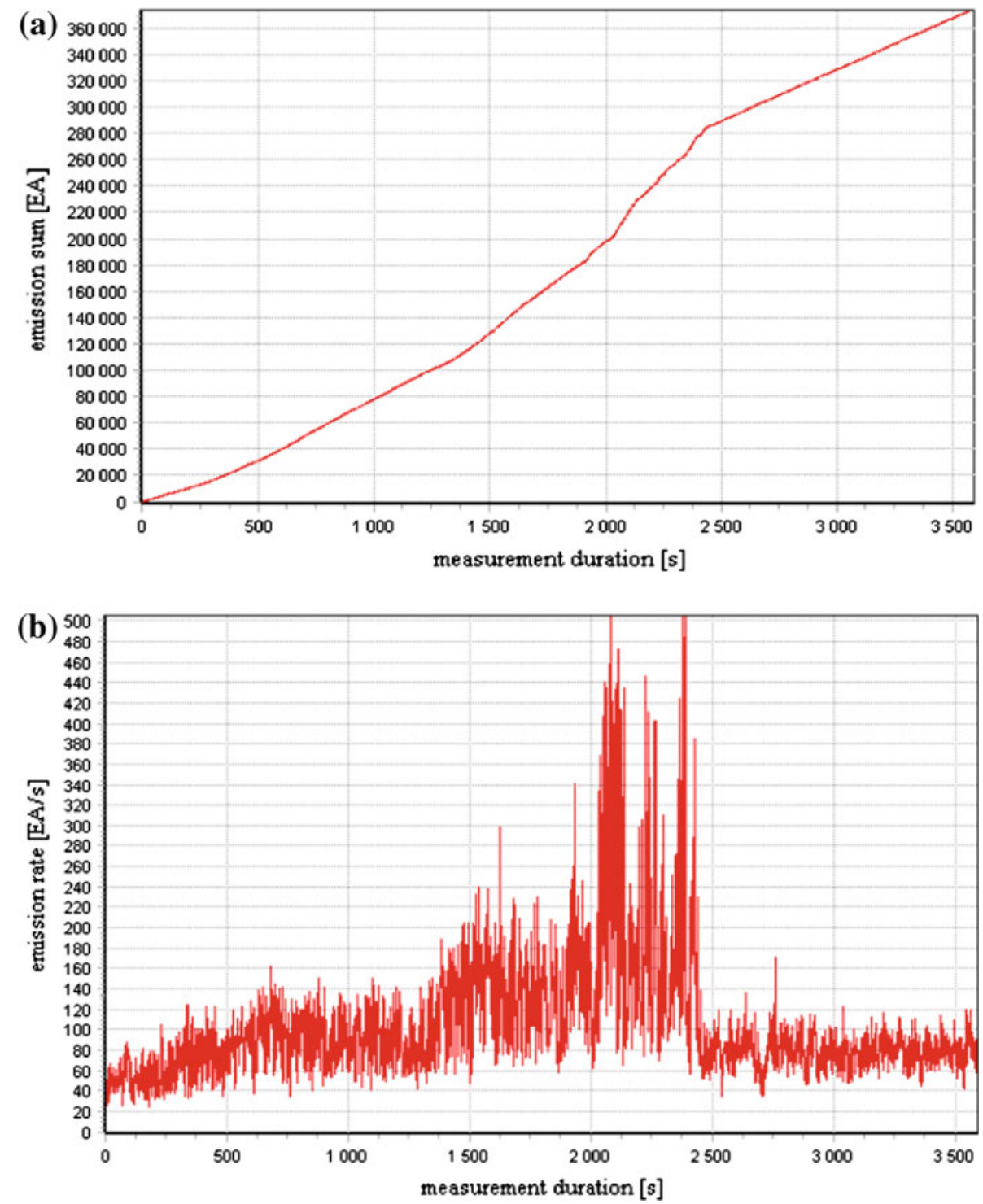

electrical treeing accompanied same qualitative changes, but failed to explicitly describe these changes quantitatively [6].

The research of electric treeing of methyl polymethacrylate or polyethylene has been carried out according to the following procedures.

Procedure 1 Once a sample is prepared (each sample was tested here only once), i.e. the needle inserted to the assumed distance in the range $0.5-2.0 \mathrm{~mm}$ from the wall covered with a conducting paint and the sample located in an oil filled transformer container, the voltage was increased up to an assumed level in the range $5-21 \mathrm{kV}$ with the rate of $0.1 \mathrm{kV} / \mathrm{s}$. For example, if the established voltage level was equal to $10 \mathrm{kV}$, its rise time was $100 \mathrm{~s}$.

The camera image and acoustic signals have been recorded within an hour or until the time of sample breakdown.
Procedure 2 Once the sample is prepared according to the Procedure 1 and located in an oil-filled transformer container, the voltage value was increased up to $5 \mathrm{kV}$ with the rate of $0.5 \mathrm{kV} / \mathrm{s}$. After $1 \mathrm{~min}$, the voltage was increased by $1 \mathrm{kV}$ with the rate of $0.1 \mathrm{kV} / \mathrm{s}$. This voltage level was kept for $1 \mathrm{~min}$ and afterwards was increased again by $1 \mathrm{kV}$ with the rate of $0.1 \mathrm{kV} / \mathrm{s}$ and such a sequence of 1 min waiting and $1 \mathrm{kV}$ voltage increase was repeated until achieving the voltage $21 \mathrm{kV}$ or until the sample breakdown.

The results of investigation of an example methyl polymethacrylate sample subject to the stresses in accordance to the Procedure 1 and the voltage of $10 \mathrm{kV}$ are shown below. Distance between the electrodes amounted to $1 \mathrm{~mm}$. Figure 4 presents a picture of the inter-electrode dielectric space of the sample. 
Fig. 6 Changes in the values of $\mathbf{a}$ the sum, and $\mathbf{b}$ the events rate of the EA signals during the electric treeing test of the sample made of methyl polymethacrylate
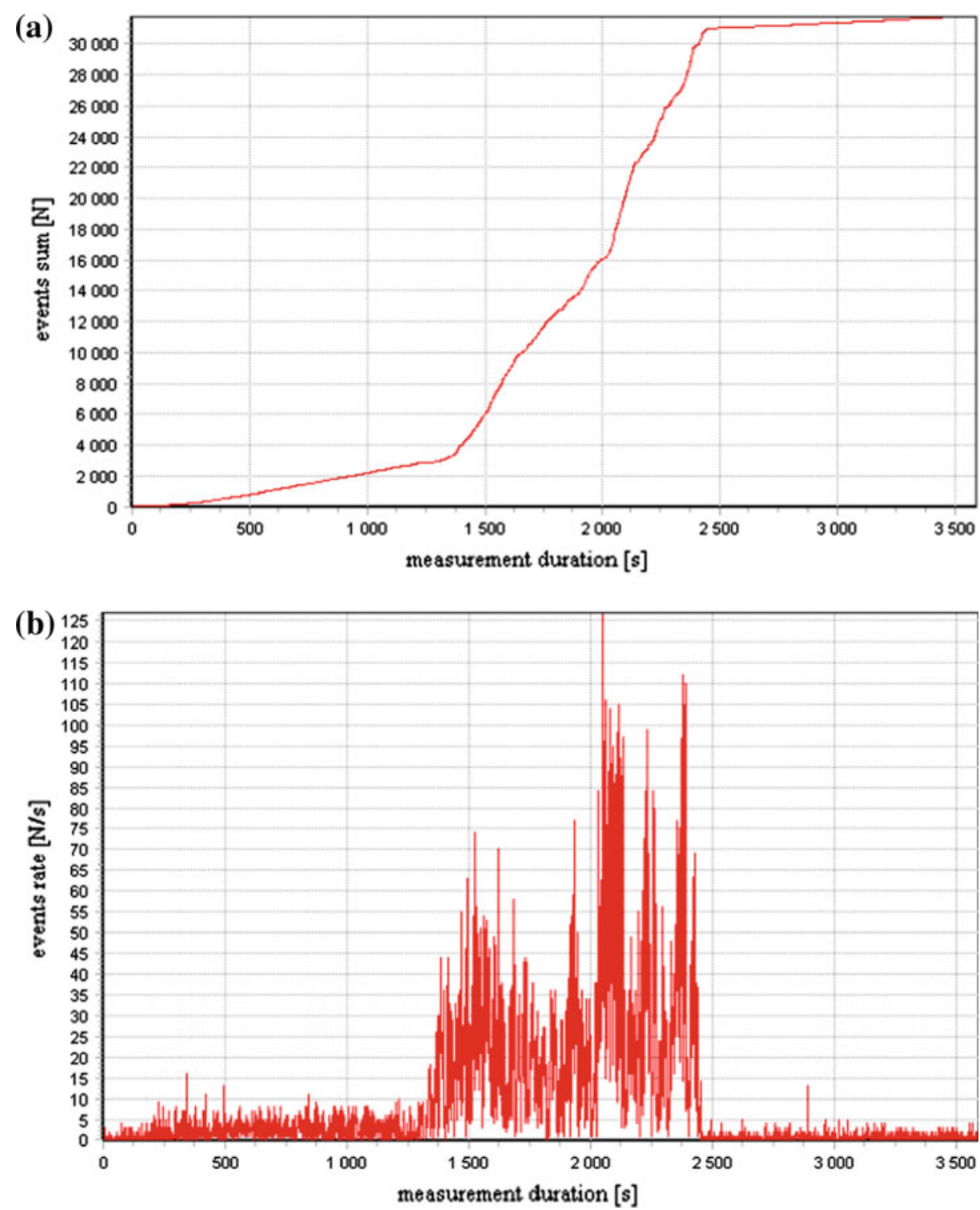

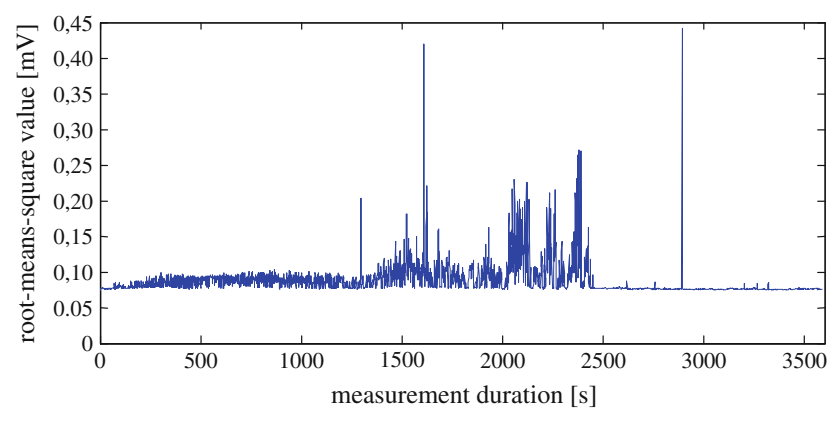

Fig. 7 Changes in root means square values of the EA signals during the electric treeing test of the sample made of methyl polymethacrylate

The photographic documentation shows that apparent treeing symptoms appeared in the 3rd minute of the measurement. Afterwards, about up to the 20th minute, the tree developed gradually, giving rise to many new branches channels. Between the 20th and 40th minute of the measurement, the tree developed mainly by extensive growth of the mesh density. Approximately, after the 40th minute, the tree development intensity was clearly reduced-no new channels appeared.

Figures 5, 6 and 7 show the time variations of the following EA descriptors, respectively: the counting sum and rate; the events sum and rate; the RMS value. On the other hand, Fig. 8 presents a frequency spectrum and spectrogram of the acoustic emission signals recorded during the measurement.

The assay allowed for easy observation of the correlation between the measured values of the EA signals descriptors and the treeing process. The first symptoms of treeing have been accompanied by the growth of the EA signals 

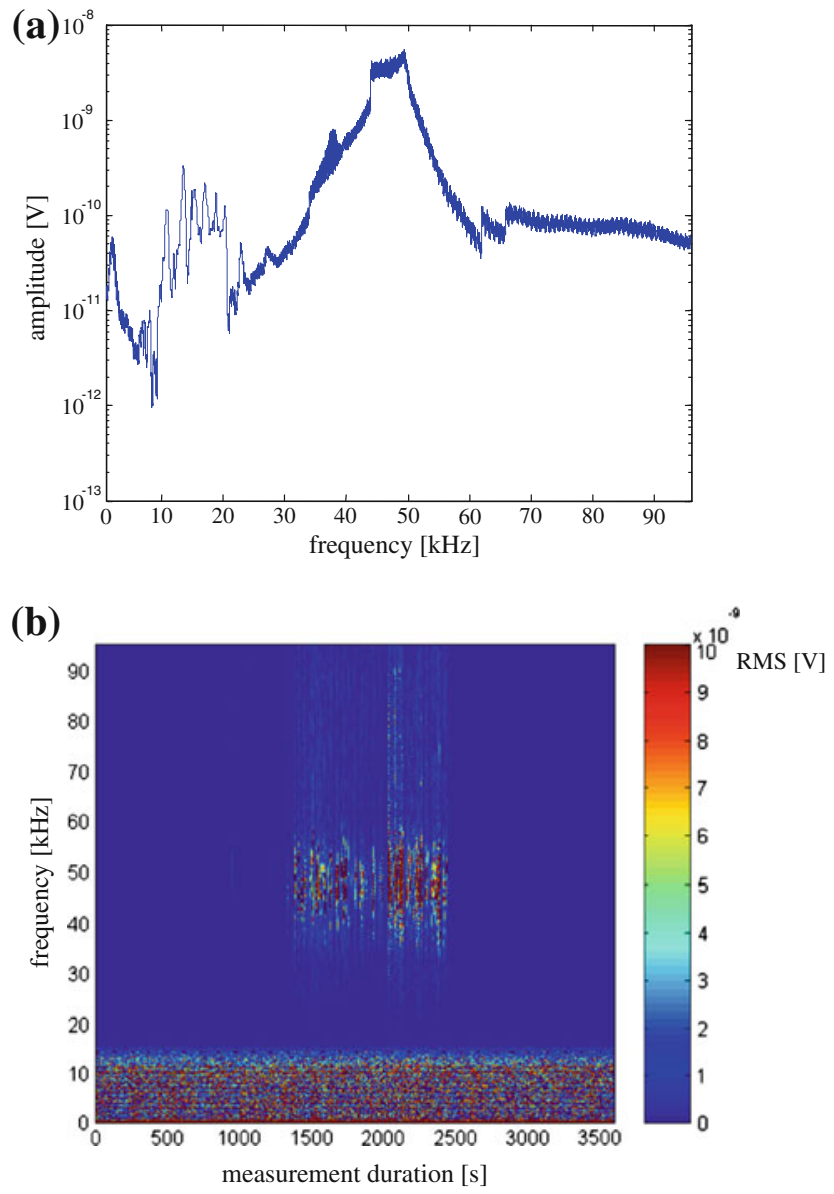

Fig. 8 a Amplitude frequency spectrum and $\mathbf{b}$ spectrogram of the EA signals during the electric treeing test of the sample made of methyl polymethacrylate

counting and the rate of EA events. During extensive growth $(1,200-2,400$ s) of density of the channels the values of these descriptors increased several times. Similar (nearly double) growth of the RMS value of EA occurred. After 2,500 s from the start of measurement, has stalled the development of the tree, thus reducing the value of descriptors to the level before the start of treeing.

In case of frequency spectrum of EA signals, the frequencies below $15 \mathrm{kHz}$ should be neglected. These signals are not related to the process of electric treeing-they come from acoustic interference [5]. Considering the above, the components in the range from 40 to $50 \mathrm{kHz}$ are at least one order higher than the others. Moreover, these components did not occur in the assays in which the treeing was not observed. Amplitudes of the above-mentioned spectrum components take maximal values for the case of extensive treeing.

Figures $9,10,11,12,13$ show typical results of the tests made with crosslinked polyethylene subject to the stress in accordance with the Procedure 1. The interelectrode distance amounted to $1.5 \mathrm{~mm}$ and the stressing voltage was equal to
$15 \mathrm{kV}$. Figure 9 presents the pictures of the interelectrode space made during the measurement. Figure 10 shows the time variations of $a$ the sum and $b$ the rate of EA signals counting, while Fig. 11a the sum and $b$ the rate of EA events. On the other hand, Fig. 12 presents the time pattern of RMS value of EA signals, and Fig. 13 shows the frequency spectrum and a spectrogram of the recorded signals.

The first symptoms of treeing appeared after about 12 minutes from the measurement start. Then the treeing developed extensively until about the 20th minute of the measurement, and afterwards reduced down. After the 40th minute density of the tree branches began to grow, nevertheless keeping a constant distance between the plane electrode and the nearest branch.

During this test in the moment of apparent treeing symptoms, a clear growth of average counting rate, events rate, and RMS EA signals values occurred. Moreover, during the initial extensive treeing period, the values of these descriptors were more than tenfold higher than in the later stage of moderate treeing. In the final stage of measurement, an apparent increase in the values of EA signal descriptors was observed (particularly the events rate and the RMS value). This was due to the EA signals of the power remarkably exceeding the former level that may be considered as a symptom of quick breakdown of the sample.

In the amplitude-frequency spectrum shown in Fig. 13a maximal amplitude values occur in the frequency components of the range $20-30 \mathrm{kHz}$. These components appear with the start of treeing that may be observed in Fig. 13b presenting a spectrogram of the EA signals. On the other hand, for a strongly intensified treeing process no particular range of the frequency components could be found. The EA signals recorded in the whole measurement range have similar large amplitudes.

In both cases of the samples made of methyl polymethacrylate and crosslinked polyethylene appearance of electric treeing was conducive to growing EA counting and events rates. Nevertheless, values of the measured descriptors, even for the same dielectric and under equal experiment conditions were remarkably different each from other. Significant differences appeared even in case of a repeated measurement performed under the same conditions, i.e. for equal inter-electrode distance and voltage values. Hence, typical values of the measured descriptors, that would discriminate various treeing forms and rates, could not be determined.

Start of treeing is accompanied by the growth of EA signals counting and events rates, provided that the process itself is relatively stable. Sometimes, in the case of a slow and uniform treeing the counting and events rates remained constant. On the other hand, in case of incidental growth of the tree, i.e. when longer periods without treeing are interrupted by short instants of treeing, the values of the above descriptors 
Fig. 9 Pictures of the interelectrode space in the crosslinked polyethylene sample: a start of the measurement, b $12 \mathrm{~min}$ after the measurement start, c 13 min after the measurement start, d 14 min after the measurement start, e 20 min after the measurement start, f 40 min after the measurement start, $\mathrm{g} 60 \mathrm{~min}$ after the measurement start
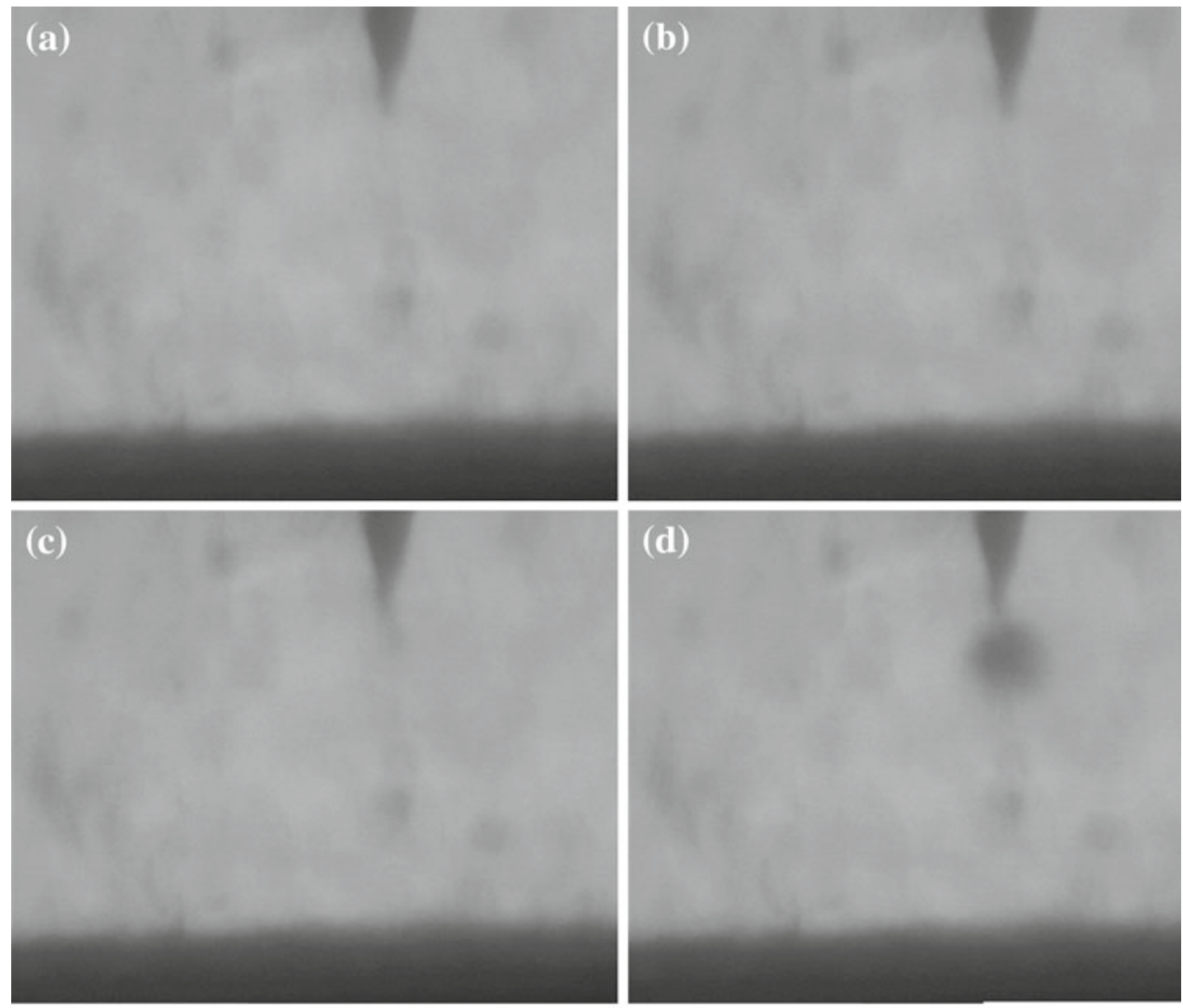

(d)
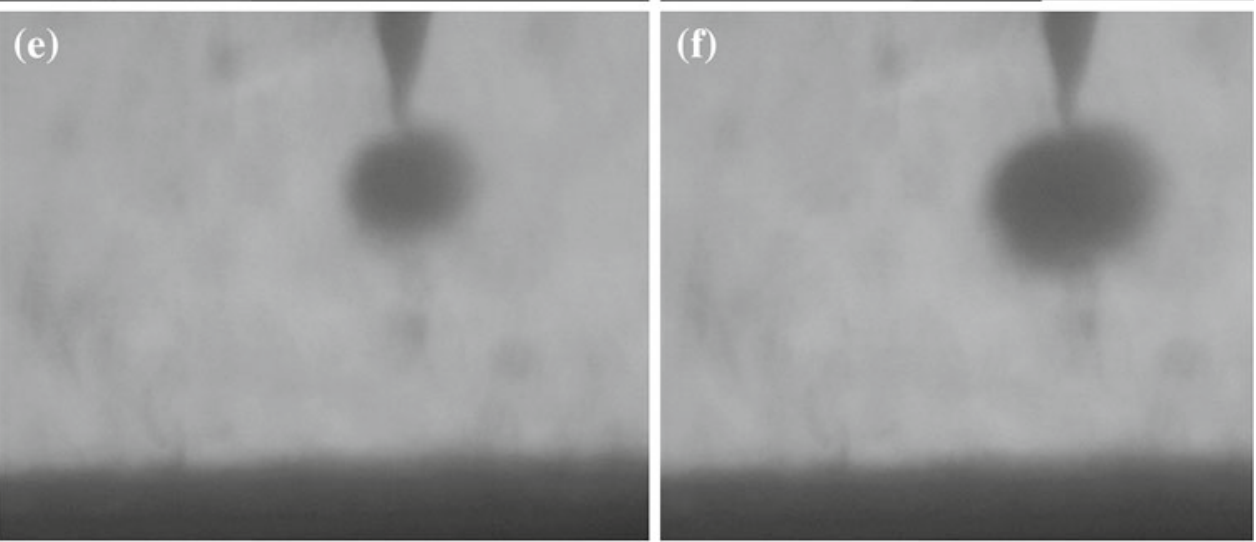

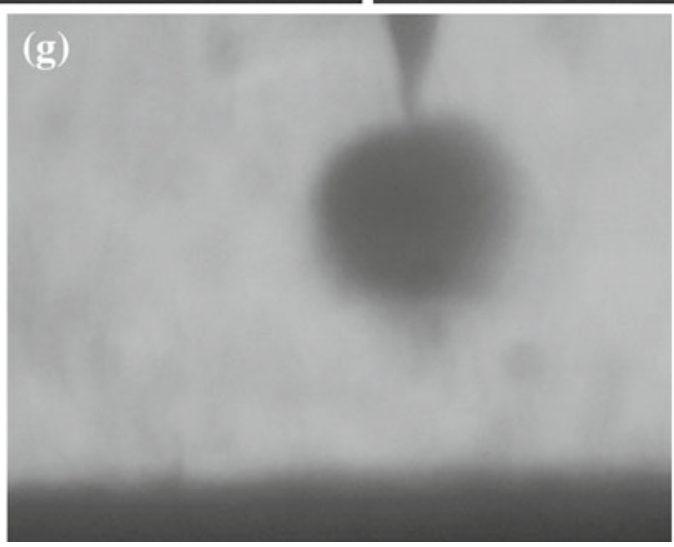


Fig. 10 Changes in the values of $\mathbf{a}$ the sum, and $\mathbf{b}$ the counting rate of the EA signals during the electric treeing test of the sample made of crosslinked polyethylene
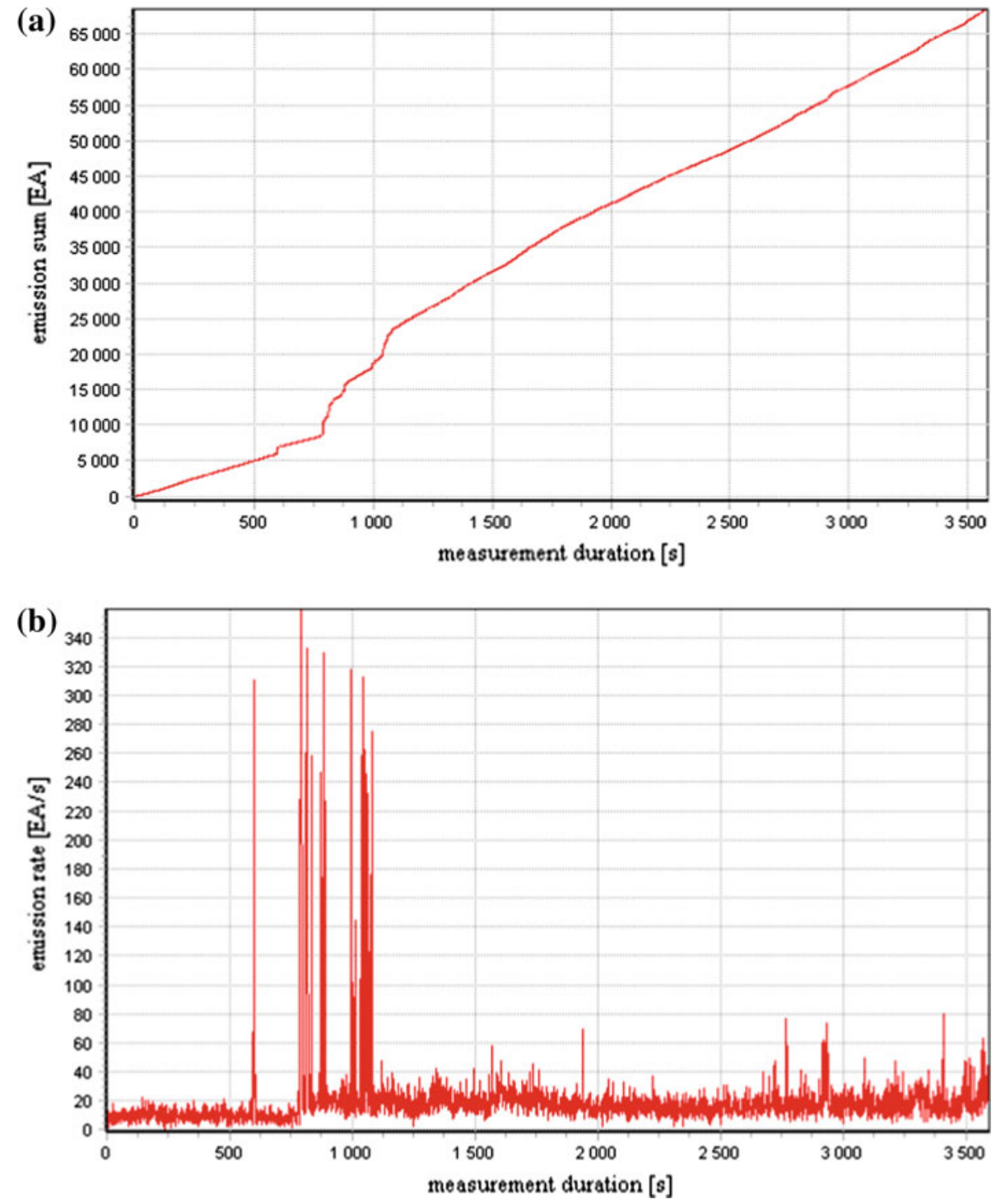

remained practically unchanged, with possible short periods of their growth (lasting no longer than several seconds).

In case of lack of treeing the time dependence of the counting and events rates of the measured EA signals have a form of straight lines beginning in the origin of the system of coordinates. Since the treeing manifestation the increments of these descriptors grow with time, provided that the treeing process is uniform. The time plots of these descriptors sometime show a bend, i.e. a segment of greater slope. In case of extensive treeing, e.g. just before the breakdown, the further part of the dependence may take a parabolic shape. Thus, sudden increase in the value gives evidence of permanent growth of the descriptor.

RMS value of EA signals remained constant provided that treeing did not occur or underwent very slowly. On the other hand, in case of more extensive treeing, even a more than ten- fold increase in the value of this descriptor occurred. This was observed both in case of instantaneous, lasting several seconds, and remarkably longer lasting tree growth processes. Hence, this descriptor may be considered as a treeing intensity factor.

The attempts to find a relationship between the values of the analyzed descriptors and the treeing intensity were unsuccessful. Similarly, no relationship was found between the values of the measured descriptors and the value of the test voltage and the value of macroscopic intensity of electric field in the dielectric [6].

Analysis of frequency distributions of the measured EA signals showed some characteristic frequency components of the EA signals that appeared while the dielectric treeing. In case of methyl polymethacrylate the treeing was accompanied by the components in the range of $40-50 \mathrm{kHz}$, while 
Fig. 11 Changes in the values of $\mathbf{a}$ the sum, and $\mathbf{b}$ the events rate of the EA signals during the electric treeing test of the sample made of crosslinked polyethylene
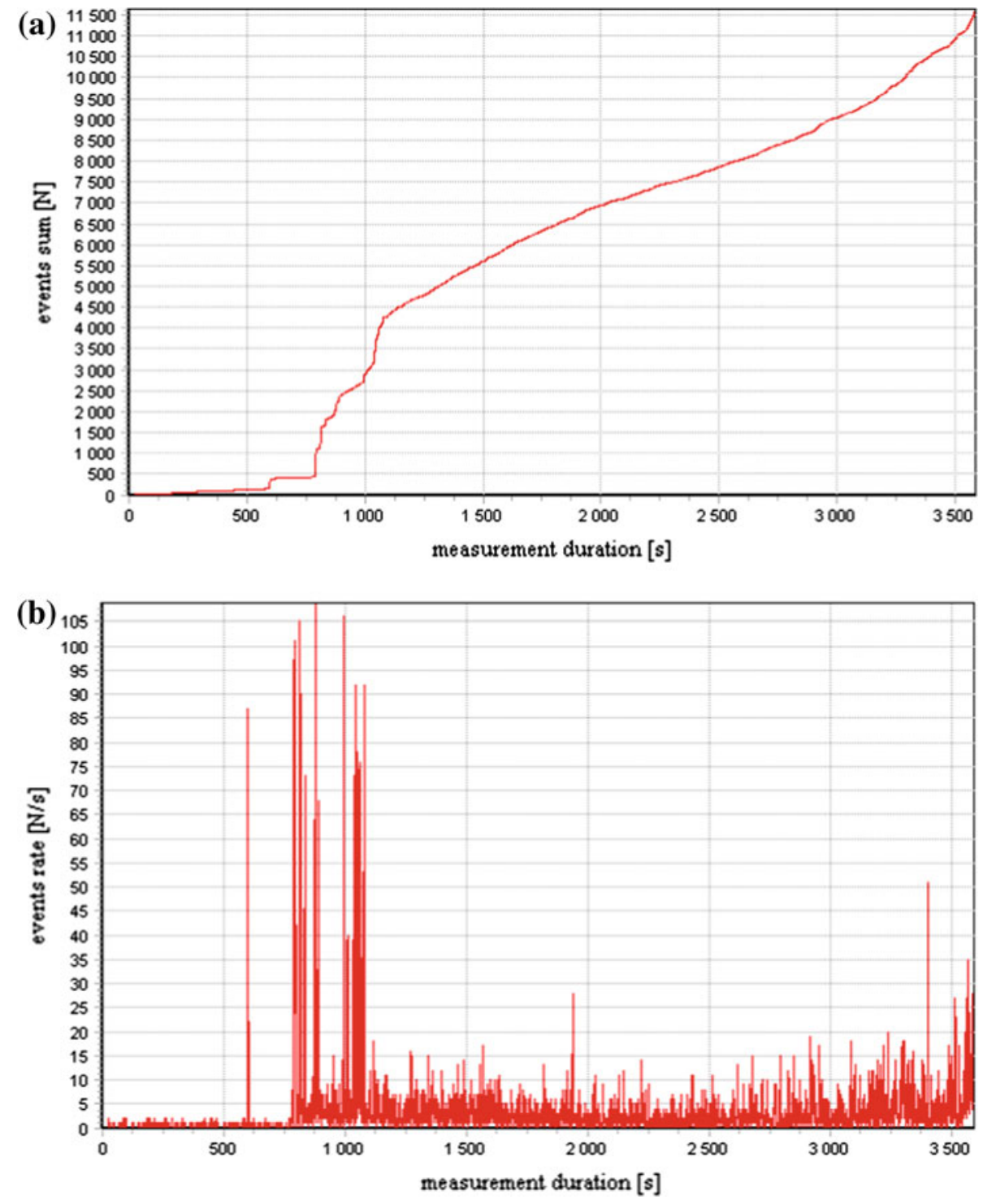

for polyethylene in the range $20-30 \mathrm{kHz}$. Moreover, frequency of the spectrum component of maximal amplitude was found. Table 1 includes specification of the results of the tests aimed at finding predominant frequencies of EA signals generated while electric treeing of methyl polymethacrylate and polyethylene.

It seems that the occurrence of dominant frequencies in the acoustic signals accompanying treeing dielectrics is a result of the resonance of pressure acoustic waves, generated by electric discharge in a channels formed in dielectric. This discharge increases temperature locally, causing the material degradation duct walls. Therefore, it is developing in the gas which is a mixture of dielectric degradation products. The composition of this gas mixture, in which there is dependent on the type of dielectric. Pressure wave propagation velocity of sound in gases, and hence the resonant frequency depends on the type of gas. It follows that the dominant frequency observed in the measured acoustic signals may be the resonance frequencies corresponding to the type of dielectric, where there is a tree.

\section{Conclusions}

Our study provides the following conclusions that are important for designers, diagnosticians, and users of high-voltage equipment including solid dielectric materials:

1. The process of electric treeing of methyl polymethacrylate and crosslinked polyethylene is accompanied by 


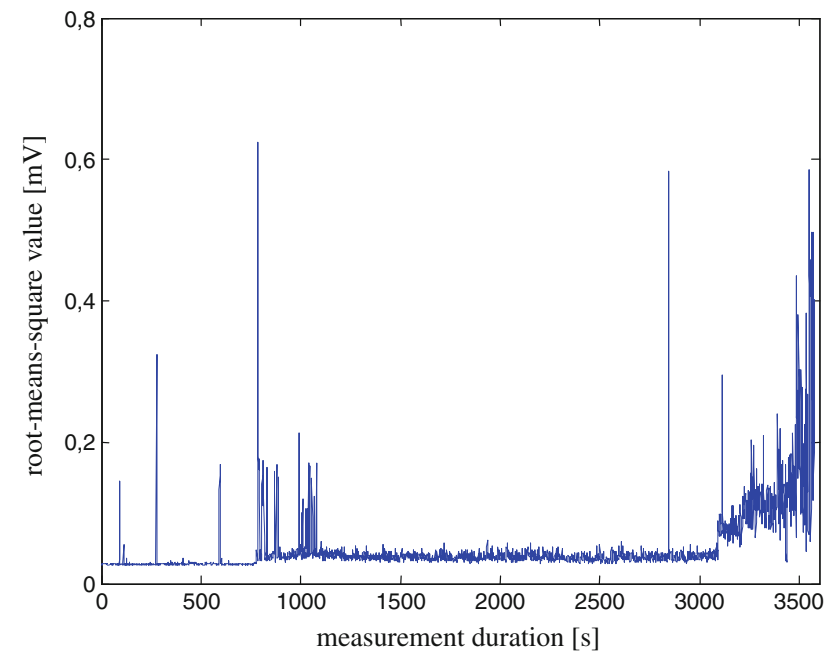

Fig. 12 Changes in root mean square values of the EA signals during the electric treeing test of the sample made crosslinked polyethylene

emission of elastic waves that may be measured and used for diagnosing the condition of the dielectric materials.

2. Initiation of electric treeing of the considered dielectric materials is accompanied by increase in the counting and events rates of the emitted acoustic signals. Moreover, the slope angle of the characteristics depicting the relationship between the sum of countings and sum of events of the EA signals during the measurement period increases.

3. The RMS value of the emitted acoustic signals is a factor of treeing intensity of the considered dielectric material. Sudden growth of treeing intensity results in significant increase in the descriptor, amounting to hundreds or even more than thousand percent.

4. Initiation of electric treeing in methyl polymethacrylate is accompanied by very high increase in amplitudes of frequency components of the EA signals in the range of $40-50 \mathrm{kHz}$. On the other hand, initiation of electric tree-
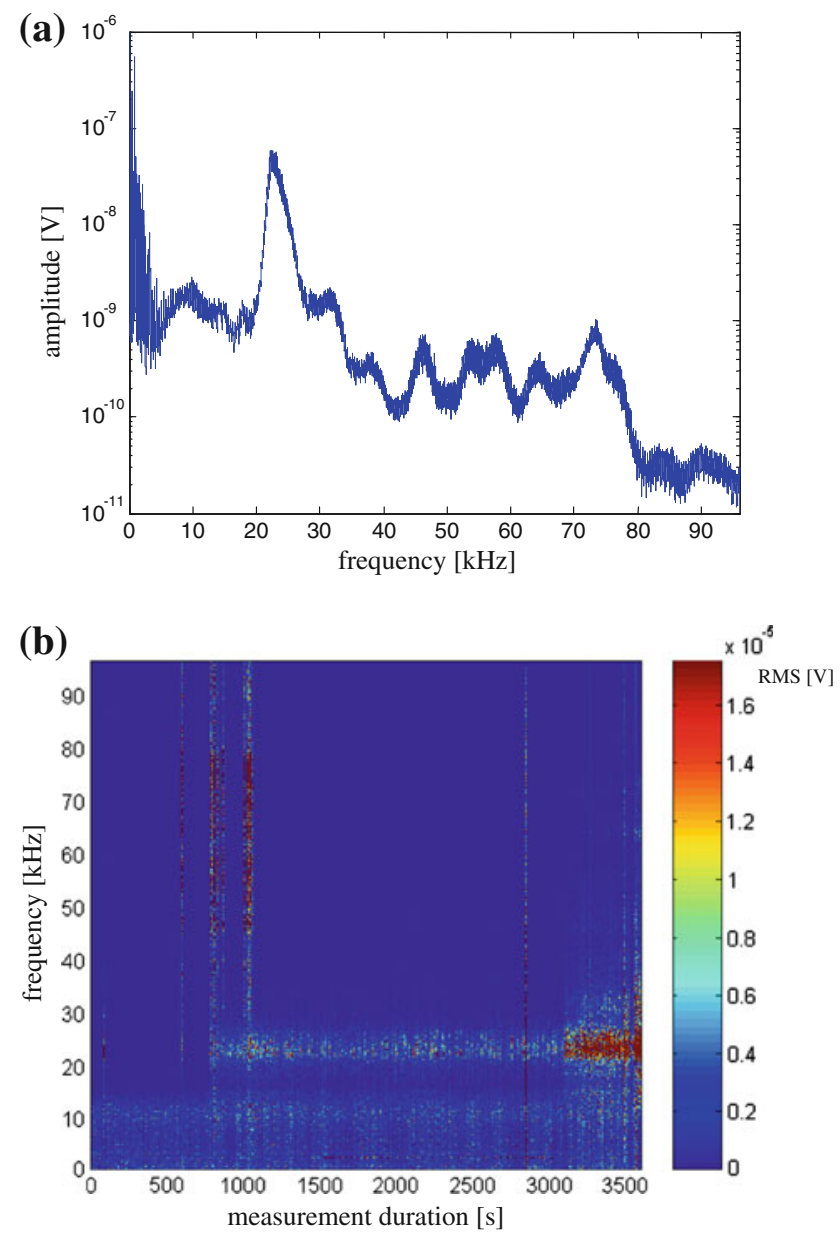

Fig. 13 a Amplitude-frequency spectrum and b spectrogram of EA signals during the electric treeing test of the sample made of crosslinked polyethylene

ing in crosslinked polyethylene is accompanied by very high increase in amplitudes of frequency components of the EA signals in the range of $20-30 \mathrm{kHz}$.
Table 1 Specification of test results of predominant frequencies of EA signals generated while electric treeing of methyl polymethacrylate and polyethylene

\begin{tabular}{|c|c|c|c|c|c|}
\hline No & Dielectric & $\begin{array}{l}\text { Frequency range } \\
\text { of maximum } \\
\text { amplitude EA } \\
\text { signals }(\mathrm{kHz})\end{array}$ & $\begin{array}{l}\text { Average value of } \\
\text { the frequency } \\
\text { component of } \\
\text { maximum } \\
\text { amplitude EA } \\
\text { signals }(\mathrm{kHz})\end{array}$ & $\begin{array}{l}\text { Standard } \\
\text { deviation of the } \\
\text { average value of } \\
\text { maximum } \\
\text { amplitude EA } \\
\text { signals }(\mathrm{kHz})\end{array}$ & $\begin{array}{l}\text { Confidence } \\
\text { interval of the } \\
\text { average value of } \\
\text { maximum } \\
\text { amplitude EA } \\
\text { signals }(\mathrm{kHz})\end{array}$ \\
\hline 1 & $\begin{array}{l}\text { Methyl poly } \\
\text { methacrylate }\end{array}$ & $36-54$ & 48.2 & 1.6 & $47.0-49.4$ \\
\hline 2 & $\begin{array}{l}\text { Crosslinked } \\
\text { polyethylene }\end{array}$ & $17-33$ & 23.1 & 2.1 & $21.7-24.6$ \\
\hline
\end{tabular}


Open Access This article is distributed under the terms of the Creative Commons Attribution Noncommercial License which permits any noncommercial use, distribution, and reproduction in any medium, provided the original author(s) and source are credited.

\section{References}

1. Białasiewicz J (2004) Wavelets and approximations. WNT, Warszawa (in Polish)

2. Ding H-Z, Varlow BR (2005) Thermodynamic model for electrical tree propagation kinetics in combined electrical and mechanical stresses. IEEE Trans Dielectr Electr Insulation 12(1):81-89

3. Dissado LA (2002) Understanding electrical trees in solids: from experiment to theory. IEEE Trans Dielectr Electr Insulation 9(4):483-497

4. Dissado LA, Dodd SJ, Champion JV, Williams PI, Alison JM (1997) Propagation of electrical tree structures in solid polymeric insulation. IEEE Trans Dielectr Electr Insulation 4(3): 259-279

5. Dobrzycki A (2008) Acoustic emission method in analysis and measurement of electrical treeing process in a strong electric field. Doctoral thesis, Poznan (in Polish)

6. Dobrzycki A, Opydo W (2007) An attempt to appraise the progress of methyl polymethacrylate degradation induced by a strong electric field on the grounds of analysis of acoustic signal emission, vol 56. Poznan University of Technology Academic Journals, Poznan, pp 197-203

7. Dobrzycki A, Opydo W, Dobroszewski R (2006) Computeraided analysis of acoustic emission signals accompanying methyl polymetacrylate degradation in a strong electric field, vol 52. Poznan University of Technology Academic Journals, Poznan, pp 201-210

8. Documentation of R3 $\alpha$ transducer. PAC, Princeton (2002)

9. Imai K (2002) Properties of tree propagation from a simulated tree channel. In: Proceedings of IEEE 2002 annual report conference on electrical insulation and dielectric phenomena. IEEE, pp 963-966

10. Kaneiwa H, Suzuoki Y, Mizutani T (2001) Characteristics of partial discharges in artificial simulated tree channels during tree propagation. IEEE Trans Dielectr Electr Insulation 8(1):843-848

11. Kaneiwa H, Suzuoki Y, Mizutani T (2000) Partial discharge characteristics and tree inception in artificial simulated tree channels. IEEE Trans Dielectr Electr Insulation 7(6):72-77

12. Kudo K (1998) Fractal analysis of electrical trees. IEEE Trans Dielectr Electr Insulation 5(5):713-726

13. Lyons RG (2006) Understanding digital signal processing. WKiE, Warszawa (in Polish)

14. Uehara H, Kudo K (2005) Temporal propagation characteristics of simulation tree considering growth probability. In: Proceedings of 2005 international symposium on electrical insulating. Kitakyushu, Japan, pp 17-20 